\title{
Property (T), finite-dimensional representations, and generic representations
}

\author{
Michal Doucha, Maciej Malicki and Alain Valette \\ Communicated by Pierre-Emmanuel Caprace
}

\begin{abstract}
Let $G$ be a discrete group with Property (T). It is a standard fact that, in a unitary representation of $G$ on a Hilbert space $\mathscr{H}$, almost invariant vectors are close to invariant vectors, in a quantitative way. We begin by showing that, if a unitary representation has some vector whose coefficient function is close to a coefficient function of some finite-dimensional unitary representation $\sigma$, then the vector is close to a sub-representation isomorphic to $\sigma$ : this makes quantitative a result of P. S. Wang. We use that to give a new proof of a result by D. Kerr, H. Li and M. Pichot, that a group $G$ with Property (T) and such that $C^{*}(G)$ is residually finite-dimensional, admits a unitary representation which is generic (i.e. the orbit of this representation in $\operatorname{Rep}(G, \mathscr{H})$ under the unitary group $U(\mathscr{H})$ is comeager). We also show that, under the same assumptions, the set of representations equivalent to a Koopman representation is comeager in $\operatorname{Rep}(G, \mathcal{H})$.
\end{abstract}

\section{Introduction}

Let $G$ be a discrete group and $\pi$ a unitary representation of $G$ on some Hilbert space $\mathscr{H}$. For a finite set $F \subset G$ and $\varepsilon>0$, a vector $\xi \in \mathscr{H}$ is $(F, \varepsilon)$-invariant if $\max _{g \in F}\|\pi(g) \xi-\xi\|<\varepsilon$. Recall that $\pi$ almost has invariant vectors if, for every pair $(F, \varepsilon)$, the group $G$ has $(F, \varepsilon)$-vectors; and that the group $G$ has Kazhdan's Property $(T)$ or is a Kazhdan group if every unitary representation of $G$ almost having invariant vectors, has non-zero invariant vectors; see e.g. [3] for Property (T). The definition can be reformulated in terms of weak containment of representations: $G$ has Property (T) if every unitary representation weakly containing the trivial representation of $G$, contains it strongly (see [3, Remark 1.1.2]). Crucial for us is an equivalent characterization due to P. S. Wang [12, Corollary 1.9 and Theorem 2.1]: the group $G$ has Property (T) if and only if for some (hence every) irreducible finite-dimensional unitary representation $\sigma$ of $G$, every unitary representation $\pi$ of $G$ that contains $\sigma$ weakly, contains it strongly.

It is a simple but useful fact that, if $G$ has Property (T) and $\pi$ is a unitary representation almost having invariant vectors, "almost invariant vectors are close to invariant vectors". More precisely, we have the following.

The first named author was supported by the GAČR project 16-34860L and RVO: 67985840 . 
Proposition 1.1 ([3, Proposition 1.1.9]). Let $G$ be a Kazhdan group. If $S$ is a finite generating set of $G$ and $\varepsilon_{0}$ is the corresponding Kazhdan constant, then for every $\delta \in] 0,1\left[\right.$ and every unitary representation $\pi$ of $G$, any $\left(S, \varepsilon_{0} \delta\right)$-invariant vector $\xi$ satisfies $\|\xi-P \xi\| \leq \delta\|\xi\|$, where $P$ is the orthogonal projection on the subspace of $\pi(G)$-invariant vectors.

For a Kazhdan group $G$ and a unitary representation $\pi$ of $G$, fix a unit vector $\xi$ and look at the coefficient function

$$
\phi_{\pi, \xi}(g)=\langle\pi(g) \xi, \xi\rangle \quad(g \in G) .
$$

The question we first address in this paper is: if $\phi_{\pi, \xi}$ is close to some coefficient of an irreducible finite-dimensional unitary representation $\sigma$ of $G$, must $\xi$ be close to a finite-dimensional invariant subspace of $\pi$ carrying a sub-representation isomorphic to $\sigma$ ? We will see that, in analogy to Proposition 1.1, the answer is positive - with some care.

Definition 1.2. Let $G$ be a finitely generated group with a symmetric finite generating set $S \subseteq G$ containing $e$ and let $\phi$ be some normalized positive definite function on $G$ associated with a unitary irreducible representation $\sigma$, of finite dimension $d$. Let $\pi$ be some unitary representation of $G$ on $\mathscr{H}$. Let $\varepsilon>0$. Say that a unit vector $\xi \in \mathscr{H}$ is $(\pi, \phi, \varepsilon)$-good if for every $s \in S^{2 d^{2}+1}$ we have $\left|\phi_{\pi, \xi}(s)-\phi(s)\right|<\varepsilon$.

Note that $S^{k}$ is just the ball of radius $k$ centered at the identity in $G$. So there is a certain lack of uniformity in Definition 1.2: we require an approximation of $\phi_{\pi, \xi}$ by $\phi$ on a ball whose size depends on the dimension of the representation $d$. Our main result, proved in Section 2, can be viewed as a quantitative version of Wang's result.

Theorem 1.3. Let $G$ be a discrete Kazhdan group, $S$ a finite symmetric generating set with $e \in S$, and let $\phi$ be a normalized positive definite function on $G$ associated with a finite-dimensional unitary irreducible representation $\sigma$ of $G$. For every $\delta \in] 0,1\left[\right.$ there exists $\varepsilon_{\phi, \delta}>0$ such that for every unitary representation $\pi$ of $G$ on a Hilbert space $\mathcal{H}$, and every unit vector $x \in \mathscr{H}$ that is $\left(\pi, \phi, \varepsilon_{\phi, \delta}\right)$-good, there exists a unit vector $x^{\prime} \in \mathcal{H}$ with $\left\|x-x^{\prime}\right\| \leq \delta$ such that the restriction of $\pi$ to the span of $\pi(G) x^{\prime}$ is isomorphic to $\sigma$.

In Section 3, we apply Theorem 1.3 to the study of the global structure of the space of unitary representations of Kazhdan groups. Let us start with the notation. Let $G$ be an arbitrary countable group and let $\mathscr{H}$ be a separable infinitedimensional Hilbert space. The set $\operatorname{Rep}(G, \mathscr{H})$ of all homomorphisms from $G$ into the unitary group $U(\mathscr{H})$ can be viewed as a closed subset of the product 
space $U(\mathscr{H})^{G}$, when we equip $U(\mathscr{H})$ with the strong operator topology. With this identification, $\operatorname{Rep}(G, \mathscr{H})$ is a Polish (i.e. separable and completely metrizable) space. We refer the reader to the work [8], especially to the section on the spaces of unitary representations, for more information about this point of view on unitary representations. Recall that two unitary representations $\pi_{1}, \pi_{2} \in \operatorname{Rep}(G, \mathscr{H})$ are isomorphic, or unitarily equivalent if there is a unitary operator $\phi \in U(\mathscr{H})$ such that $\pi_{1}(g)=\phi \pi_{2}(g) \phi^{*}$ for every $g \in G$. Notice that this is an orbit equivalence relation given by the action of the unitary group $U(\mathscr{H})$ on the space $\operatorname{Rep}(G, \mathscr{H})$ by conjugation. Kechris raised the question (see again the section on the space of unitary representations in [8]) of whether or not there are countable groups with a generic unitary representation, where "generic" here means its conjugacy class is large in the sense of Baire category, i.e. a representation whose class under the unitary equivalence contains a dense $G_{\delta}$ subset. As a matter of fact, we note that it follows from the topological zero-one law that for every countable group $G$ either

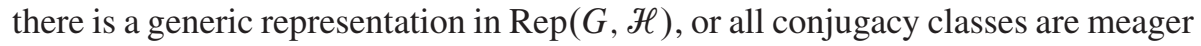
(see e.g. [7, Theorem 8.46]; to apply it, note that there is a dense conjugacy class in $\operatorname{Rep}(G, \mathscr{H})$ - indeed, take some countable dense set of representations from $\operatorname{Rep}(G, \mathscr{H})$ and consider their direct sum).

Here as an application of Theorem 1.3 we prove the following result.

Theorem 1.4. Let $G$ be a discrete Kazhdan group such that finite-dimensional representations are dense in the unitary dual $\hat{G}$. Then there is a generic unitary representation of $G$.

We note that, although not explicitly stated there, this result already follows from a more general result of Kerr, Li and Pichot from [9], where they prove (see Theorem 2.5 there) that if $A$ is a separable $C^{*}$-algebra where finite-dimensional representations are dense in $\hat{A}$, then there is a dense $G_{\delta}$ class in $\operatorname{Rep}(A, \mathcal{H})$. Theorem 1.4 is then a special case for $A=C^{*}(G)$. Our proof is nevertheless done by more elementary means, in particular it does not invoke Voiculescu's theorem (see [9, proof of Theorem 2.5] for details).

The converse of Theorem 1.4 can be also derived from [9], see Remark 3.1 below.

Another open question posed by Kechris in [8, Problem H.16] is whether the subset of those representations $\pi \in \operatorname{Rep}(G, \mathscr{H})$, where $G$ is still a countable group, that are equivalent to Koopman representations, is meager in $\operatorname{Rep}(G, \mathcal{H})$ or not. Such representations are called realizable by an action in [8]. Let us recall the terminology first. Let $(X, \mu)$ be a standard probability space (i.e. a space isomorphic to the unit interval $[0,1]$ equipped with the Lebesgue measure). Let $\alpha: G \curvearrowright(X, \mu)$ be an action of a countable group $G$ on $X$ by measure-preserving measurable 
transformations. Consider the unitary representation $\pi_{\alpha}: G \rightarrow L^{2}(X, \mu)$ defined by $\pi_{\alpha}(g) f(x)=f\left(\alpha\left(g^{-1}, x\right)\right)$ for every $f \in L^{2}(X, \mu)$. The Koopman representation of $\alpha$ is the restriction of $\pi_{\alpha}$ to the invariant subspace $L_{0}^{2}(X, \mu)$, which is the orthogonal complement of the invariant subspace of constant functions.

In Section 4 we prove the following result addressing the question of Kechris.

Theorem 1.5. Let $G$ be a discrete Kazhdan group such that finite-dimensional representations are dense in the unitary dual $\hat{G}$. Then the set of representations realizable by an action is comeager in $\operatorname{Rep}(G, \mathscr{H})$.

Let us record that the condition that finite-dimensional representations are dense in the unitary dual $\hat{G}$ is, by the result of Archbold from [1], equivalent with the statement that the full group $C^{*}$-algebra $C^{*}(G)$ is residually finite-dimensional. That is in turn, by the result of Exel and Loring from [6] (see also [11]), equivalent with the statement that finite-dimensional representations are dense in $\operatorname{Rep}(G, \mathscr{H})$, which we shall use in the proof. Note that we call a representation $\pi \in \operatorname{Rep}(G, \mathscr{H})$ finite-dimensional if the subalgebra $\pi(G)$ generates in $B(\mathscr{H})$ is finite-dimensional.

The existence of infinite discrete Kazhdan groups with residually finite-dimensional $C^{*}$-algebras seems to be open - see [3, Question 7.10] and also Lubotzky and Shalom [10, Question 6.5] where they ask if there are infinite discrete Kazhdan groups with property FD, which is strictly stronger than having a residually finitedimensional $C^{*}$-algebra (a group has property $F D$ if representations factoring through finite groups are dense in the unitary dual).

Question 1.6. It is known that being residually finite is not a sufficient condition to have a residually finite-dimensional $C^{*}$-algebra by a result of Bekka [2]. However how about being LERF? (Recall that a finitely generated group is LERF if any finitely generated subgroup is the intersection of the finite index subgroups containing it.) Ershov and Jaikin-Zapirain constructed in [5] a Kazhdan group which is LERF. Is its group $C^{*}$-algebra residually finite-dimensional?

Remark 1.7. We note that on the other hand we cannot exclude the possibility of proving by a different argument that for every infinite group $G$, all classes in $\operatorname{Rep}(G, \mathscr{H})$ are meager. That would together with Theorem 1.4 give that there are no infinite Kazhdan groups with a residually finite-dimensional $C^{*}$-algebra.

\section{A quantitative version of Wang's theorem}

Let $G$ be an infinite, finitely generated group. Let $S$ be a finite, symmetric, generating set of $G$, with $e \in S$. Let $\mathbb{C} G$ be the complex group ring of $G$. 


\subsection{Quantifying the Burnside theorem}

Let $\sigma$ be an irreducible unitary representation of dimension $d$, i.e. a homomorphism $\sigma: G \rightarrow U_{d}(\mathbb{C})$ such that $\sigma(G)$ has no proper invariant subspace. The classical Burnside theorem says that $\sigma(\mathbb{C} G)=M_{d}(\mathbb{C})$, i.e. $\sigma(G)$ contains a basis of $M_{d}(\mathbb{C})$.

Definition 2.1. Set $k(\sigma)=\min \left\{k>0: \operatorname{dim}_{\mathbb{C}} \operatorname{span} \sigma\left(S^{k}\right)=d^{2}\right\}$.

Lemma 2.2. There is a constant $C>0$ (only depending on $S$ ) such that

$$
C \log d \leq k(\sigma) \leq d^{2} .
$$

Proof. We have

$$
d^{2}=\operatorname{dim}_{\mathbb{C}} \operatorname{span} \sigma\left(S^{k(\sigma)}\right) \leq\left|\sigma\left(S^{k(\sigma)}\right)\right| \leq\left|S^{k(\sigma)}\right| \leq|S|^{k(\sigma)} .
$$

Taking logarithms,

$$
\frac{2}{\log |S|} \log d \leq k(\sigma) .
$$

To prove the upper bound, observe that the sequence $\operatorname{span} \sigma\left(S^{k}\right)$ of subspaces of $M_{d}(\mathbb{C})$ is strictly increasing for $k<k(\sigma)$. Indeed, assume that $k$ is such that $\operatorname{span} \sigma\left(S^{k}\right)=\operatorname{span} \sigma\left(S^{k+1}\right)$ : this means that $\operatorname{span} \sigma\left(S^{k}\right)$ is stable by left multiplication by $\sigma(S)$, hence by $\sigma(G)$ as $S$ is generating. Since the identity matrix is in $\sigma\left(S^{k}\right)$, we have

$$
\sigma(G) \subset \operatorname{span} \sigma\left(S^{k}\right),
$$

hence $k \geq k(\sigma)$. From this it is clear that $k(\sigma) \leq d^{2}$.

Let $v$ be a unit vector in $\mathbb{C}^{d}$. Since $v$ is cyclic for $\sigma(G)$, the map

$$
T_{v}: \mathbb{C} S^{k(\sigma)} \rightarrow \mathbb{C}^{d}, \quad f \mapsto \sigma(f) v
$$

is onto. Let $\left(\operatorname{ker} T_{v}\right)^{\perp}$ denote the orthogonal of $\operatorname{ker} T_{v}$ in $\mathbb{C} S^{k(\sigma)}$ and let $U_{v}$ be the

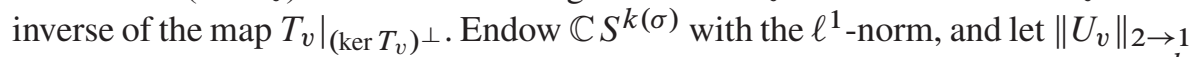
be the corresponding operator norm of $U_{v}$. So for every $w$ a unit vector in $\mathbb{C}^{d}$, there exists $f \in \mathbb{C} S^{k(\sigma)}$ with $\|f\|_{1} \leq\left\|U_{v}\right\|_{2 \rightarrow 1}$ such that $\sigma(f) v=w$.

Lemma 2.3. There exists $M>0$ such that for every two unit vectors $v, w \in \mathbb{C}^{d}$, there exists $f \in \mathbb{C} S^{k(\sigma)}$ with $\|f\|_{1} \leq M$ such that $\sigma(f) v=w$.

Proof. This is the preceding observation plus compactness of the unit sphere in $\mathbb{C}^{d}$ : the constant $M=\max _{\|v\|=1}\left\|U_{v}\right\|_{2 \rightarrow 1}$ does the job. 


\subsection{From weak containment to weak containment à la Zimmer}

Recall that, if $\pi, \rho$ are unitary representations of a discrete group $G$, the representation $\pi$ is weakly contained in the representation $\rho$ (i.e. $\pi \preceq \rho$ ) if every function of positive type associated with $\pi$ can be pointwise approximated by finite sums of positive definite type associated with $\rho$. If $\pi$ is irreducible, this is equivalent to requiring that every normalized function of positive type associated with $\pi$ can be pointwise approximated by normalized functions of positive type associated with $\rho$ (see [3, Proposition F.1.4]).

Zimmer introduced in [13, Definition 7.3.5] a different, inequivalent notion of weak containment. A $n \times n$-submatrix of $\pi$ is a function

$$
G \rightarrow M_{n}(\mathbb{C}), \quad g \mapsto\left(\left\langle\pi(g) e_{i}, e_{j}\right\rangle\right)_{1 \leq i, j \leq n},
$$

where $\left\{e_{1}, \ldots, e_{n}\right\}$ is an orthonormal family in $\mathscr{H}_{\pi}$. Say that $\pi$ is weakly contained in $\rho$ in Zimmer's sense (i.e. $\pi \preceq Z \rho$ ) if, for every $n>0$, every $n \times n$-submatrix of $\pi$ can be pointwise approximated by $n \times n$-submatrices of $\rho$. The exact relation with the classical notion recalled above, is worked out in [3, Remark F.1.2 (ix)]; in particular, when $\pi$ is irreducible, $\pi \preceq \rho$ implies $\pi \preceq Z \rho$. Our first goal will be to make the latter statement quantitative. For this we need a definition.

Let $\phi$ be associated with $\sigma$, as in Definition 1.2. Let $v$ be a unit vector in $\mathscr{H}_{\sigma}$ such that $\phi=\phi_{\sigma, v}$. Let $e_{1}, \ldots, e_{d}$ be an orthonormal basis of $\mathbb{C}^{d}$; by Lemma 2.3, we find functions $f_{1}, \ldots, f_{d} \in \mathbb{C} S^{k(\sigma)}$ with $\max _{i}\left\|f_{i}\right\|_{1} \leq M$ such that

$$
\sigma\left(f_{i}\right) v=e_{i} \quad(i=1, \ldots, d) .
$$

Lemma 2.4. Let $\pi \in \operatorname{Rep}(G, \mathscr{H})$ be a unitary representation. Assume that there is $\varepsilon>0$ and a unit vector $\eta \in \mathscr{H}$ such that for $s \in S^{2 k(\sigma)+1}$ we have

$$
|\langle\pi(s) \eta, \eta\rangle-\langle\sigma(s) v, v\rangle|<\varepsilon .
$$

Set $\eta_{i}=\pi\left(f_{i}\right) \eta$. Then for $i, j=1, \ldots, d$ and $g \in S$,

$$
\left|\left\langle\sigma(g) e_{i}, e_{j}\right\rangle-\left\langle\pi(g) \eta_{i}, \eta_{j}\right\rangle\right| \leq \varepsilon M^{2} .
$$

Proof. For $g \in S$,

$$
\begin{aligned}
& \left|\left\langle\sigma(g) e_{i}, e_{j}\right\rangle-\left\langle\pi(g) \eta_{i}, \eta_{j}\right\rangle\right| \\
& \quad=\left|\left\langle\sigma(g) \sigma\left(f_{i}\right) v, \sigma\left(f_{j}\right) v\right\rangle-\left\langle\pi(g) \pi\left(f_{i}\right) \eta, \pi\left(f_{j}\right) \eta\right\rangle\right| \\
& \quad=\left|\sum_{s, t \in G} f_{i}(s) \overline{f_{j}(t)}\left(\left\langle\sigma\left(t^{-1} g s\right) v, v\right\rangle-\left\langle\pi\left(t^{-1} g s\right) \eta, \eta\right\rangle\right)\right| \\
& \quad \leq \sum_{s, t \in G}\left|f_{i}(s)\right|\left|f_{j}(s)\right|\left|\left\langle\sigma\left(t^{-1} g s\right) v, v\right\rangle-\left\langle\pi\left(t^{-1} g s\right) \eta, \eta\right\rangle\right| .
\end{aligned}
$$


Since the supports of the functions $f_{i}$ are contained in $S^{k(\sigma)}$, and since we have $t^{-1} g s \in S^{2 k(\sigma)+1}$ for $s, t \in S^{k(\sigma)}$, we find, using the assumption, that

$$
\left|\left\langle\sigma(g) e_{i}, e_{j}\right\rangle-\left\langle\pi(g) \eta_{i}, \eta_{j}\right\rangle\right| \leq \varepsilon \sum_{s, t \in G}\left|f_{i}(s)\left\|f_{j}(t) \mid=\varepsilon\right\| f_{i}\left\|_{1}\right\|_{j} \|_{1} \leq \varepsilon M^{2}\right.
$$

as desired.

In the previous proof, by applying the Gram-Schmidt orthonormalization process to the $\eta_{i}$, it is possible to show that the $d \times d$-submatrix $\left(\left\langle\sigma(\cdot) e_{i}, e_{j}\right\rangle\right)_{1 \leq i, j \leq d}$ of $\sigma$ is close on $S$ to some $d \times d$-submatrix of $\alpha$, with an explicit bound; but we do not need that fact at this point.

\subsection{Quantifying Wang's theorem}

Let $\mathscr{H}_{\sigma}$ be the ( $d$-dimensional) Hilbert space of $\sigma$, and let $\mathscr{H}_{\bar{\sigma}}$ be the conjugate Hilbert space (with complex conjugate scalar multiplication and complex conjugate inner product), equipped with the conjugate representation $\bar{\sigma}$. Form the tensor product $\mathscr{H}_{\bar{\sigma}} \otimes \mathscr{H}_{\pi}$, carrying the representation $\bar{\sigma} \otimes \pi$. Set $\xi_{i}=e_{i} \otimes \eta_{i}$ and $\xi=\sum_{i=1}^{d} \xi_{i} \in \mathscr{H}_{\bar{\sigma}} \otimes \mathscr{H}_{\pi}$, where the $e_{i}$ and $\eta_{i}$ are as in the section above; observe that the $\xi_{i}$ are pairwise orthogonal. We need an estimate on how $\xi$ is moved by $\bar{\sigma} \otimes \pi$ :

$$
\begin{aligned}
\|\xi-(\bar{\sigma} \otimes \pi)(g) \xi\|^{2} & =2\|\xi\|^{2}-2 \operatorname{Re}\langle(\bar{\sigma} \otimes \pi)(g) \xi, \xi\rangle \\
& =2 \sum_{i=1}^{d}\left\|\xi_{i}\right\|^{2}-2 \sum_{i, j=1}^{d} \operatorname{Re}\left\langle(\bar{\sigma} \otimes \pi)(g) \xi_{i}, \xi_{j}\right\rangle \\
& =2 \sum_{i=1}^{d}\left\|\eta_{i}\right\|^{2}-2 \sum_{i, j=1}^{d} \operatorname{Re}\left\langle e_{j}, \sigma(g) e_{i}\right\rangle\left\langle\pi(g) \eta_{i}, \eta_{j}\right\rangle .
\end{aligned}
$$

Observe that for every $g \in G$ we have

$$
d=\sum_{i, j=1}^{d}\left\langle\sigma(g) e_{i}, e_{j}\right\rangle\left\langle e_{j}, \sigma(g) e_{i}\right\rangle
$$

as the $e_{i}$ form an orthonormal basis. Subtracting and adding $2 d$ to the previous formula, we get

$$
\begin{aligned}
\|\xi-(\bar{\sigma} \otimes \pi)(g) \xi\|^{2} \\
\quad=2\left[\sum_{i=1}^{d}\left(\left\|\eta_{i}\right\|^{2}-1\right)\right]-2 \sum_{i, j=1}^{d} \operatorname{Re}\left\langle e_{j}, \sigma(g) e_{i}\right\rangle\left(\left\langle\pi(g) \eta_{i}, \eta_{j}\right\rangle-\left\langle\sigma(g) e_{i}, e_{j}\right\rangle\right)
\end{aligned}
$$


hence, using Cauchy-Schwarz,

$$
\begin{aligned}
& \|\xi-(\bar{\sigma} \otimes \pi)(g) \xi\|^{2} \\
& \quad \leq 2 \sum_{i=1}^{d}\left|\left\|\eta_{i}\right\|^{2}-1\right|+2 \sum_{i, j=1}^{d}\left|\left\langle\pi(g) \eta_{i}, \eta_{j}\right\rangle-\left\langle\sigma(g) e_{i}, e_{j}\right\rangle\right| .
\end{aligned}
$$

Theorem 1.3 will follow immediately form the next proposition, together with Lemma 2.2

Proposition 2.5. Let $G$ be a discrete Kazhdan group, $S$ a finite symmetric generating set with $e \in S$, and let $\phi$ be a normalized positive definite function on $G$ associated with a finite-dimensional unitary irreducible representation $\sigma$ of $G$. For every $\delta \in] 0,1\left[\right.$ there exists $\varepsilon_{\phi, \delta}>0$ such that for every $\pi \in \operatorname{Rep}(G, \mathscr{H})$, and every unit vector $x \in \mathscr{H}$ such that $\left|\phi(s)-\phi_{\pi, x}(s)\right|<\varepsilon_{\phi, \delta}$ for $s \in S^{2 k(\sigma)+1}$, there exists a unit vector $x^{\prime} \in \mathscr{H}$ with $\left\|x-x^{\prime}\right\| \leq \delta$ such that the restriction of $\pi$ to the span of $\pi(G) x^{\prime}$ is isomorphic to $\sigma$.

Proof. Set $d=\operatorname{dim} \sigma$. Let $v$ be a unit vector in $\mathscr{H}_{\sigma}$ such that $\phi(g)=\langle\sigma(g) v, v\rangle$ for every $g \in G$. As in Section 2.2, for an orthonormal basis $e_{1}, \ldots, e_{d}$ of $\mathbb{C}^{d}$, we find functions $f_{1}, \ldots, f_{d} \in \mathbb{C} S^{k(\sigma)}$ with $\max _{i}\left\|f_{i}\right\|_{1} \leq M$ such that

$$
\sigma\left(f_{i}\right) v=e_{i} \quad(i=1, \ldots, d) .
$$

Let $0<\varepsilon_{0}<2$ be such that $\left(S, \varepsilon_{0}\right)$ is a Kazhdan pair for $G$. Fix $\left.\delta \in\right] 0,1[$, and set

$$
\varepsilon_{\phi, \delta}=\varepsilon=\frac{\delta^{2} \varepsilon_{0}^{2}}{24 d(d+1) M^{2}} .
$$

Let $\pi \in \operatorname{Rep}(G, \mathscr{H})$ and let $x \in \mathscr{H}$ be a unit vector with $\left|\phi_{\pi, x}(s)-\phi(s)\right|<\varepsilon$ for $s \in S^{2 k(\sigma)+1}$. Set $\eta_{i}=\pi\left(f_{i}\right) x$. We may assume that $e_{1}=v$ and the function $f_{1}$ is $\delta_{e}$, so that $\eta_{1}=x$. We want to prove that the vector

$$
\xi=\sum_{i=1}^{d}\left(e_{i} \otimes \eta_{i}\right) \in \mathscr{H}_{\bar{\sigma}} \otimes \mathscr{H}
$$

is $\left(S, t \varepsilon_{0}\right)$-invariant for some $0<t<1$, in order to apply Proposition 1.1.

For $g \in S$ we have, by Lemma 2.4 and inequality (2.1),

$$
\|\xi-(\bar{\sigma} \otimes \pi)(g) \xi\|^{2} \leq 2 d \varepsilon M^{2}+2 d^{2} \varepsilon M^{2}=2 d(d+1) \varepsilon M^{2}=\frac{\delta^{2} \varepsilon_{0}^{2}}{12} .
$$

Again by Lemma 2.4, evaluated at $g=e$, we have

$$
\left|\left\|\eta_{i}\right\|^{2}-1\right| \leq \varepsilon M^{2}<\frac{1}{2}
$$


hence $\frac{1}{2} \leq\left\|\eta_{i}\right\|^{2} \leq \frac{3}{2}$ and $\frac{d}{2} \leq\|\xi\|^{2}=\sum_{i=1}^{d}\left\|\eta_{i}\right\|^{2} \leq \frac{3 d}{2}$. So that, for $g \in S$,

$$
\|\xi-(\bar{\sigma} \otimes \pi)(g) \xi\|^{2} \leq \frac{\delta^{2} \varepsilon_{0}^{2}}{6 d}\|\xi\|^{2} .
$$

By Proposition 1.1, there exists a $G$-fixed $\xi^{\prime} \in \mathscr{H}_{\bar{\sigma}} \otimes \mathscr{H}$ such that

$$
\left\|\xi-\xi^{\prime}\right\|^{2} \leq \frac{\delta^{2}}{6 d}\|\xi\|^{2} \text {. }
$$

Write $\xi^{\prime}=\sum_{i=1}^{d} e_{i} \otimes \zeta_{i}$, so that

$$
\left\|\xi-\xi^{\prime}\right\|^{2}=\sum_{i=1}^{d}\left\|\eta_{i}-\zeta_{i}\right\|^{2} .
$$

Identify $\mathscr{H}_{\bar{\sigma}} \otimes \mathscr{H}$ with the space of linear operators from $\mathscr{H}_{\sigma}$ to $\mathscr{H}$ (endowed with the Hilbert-Schmidt norm), via $u \otimes y \mapsto(w \mapsto\langle w, u\rangle y)$. Then $\xi^{\prime}$ identifies with the operator $w \mapsto \sum_{i=1}^{d}\left\langle w, e_{i}\right\rangle \zeta_{i}$, which is therefore an intertwining operator between $\sigma$ and $\pi$. The image of this operator, which is $\operatorname{span}\left\{\zeta_{1}, \ldots, \zeta_{d}\right\}$, carries a sub-representation of $\pi$ unitarily equivalent to $\sigma$ (by Schur's lemma). Set $x^{\prime \prime}=\zeta_{1}$. Then

$$
\begin{aligned}
\left\|x-x^{\prime \prime}\right\|^{2} & =\left\|\eta_{1}-\zeta_{1}\right\|^{2} \leq \sum_{i=1}^{d}\left\|\eta_{i}-\zeta_{i}\right\|^{2}=\left\|\xi-\xi^{\prime}\right\|^{2} \\
& \leq \frac{\delta^{2}}{6 d}\|\xi\|^{2} \leq \frac{\delta^{2}}{6 d} \frac{3 d}{2}=\frac{\delta^{2}}{4},
\end{aligned}
$$

i.e. $\left\|x-x^{\prime \prime}\right\| \leq \frac{\delta}{2}$. Finally, set $x^{\prime}=\frac{x^{\prime \prime}}{\left\|x^{\prime \prime}\right\|}$, a unit vector in $\mathscr{H}$. Then by the triangle inequality,

$$
\begin{aligned}
\left\|x-x^{\prime}\right\| & \leq\left\|x-x^{\prime \prime}\right\|+\left\|x^{\prime \prime}-x^{\prime}\right\|=\left\|x-x^{\prime \prime}\right\|+\left\|x^{\prime \prime}\right\|\left|1-\frac{1}{\left\|x^{\prime \prime}\right\|}\right| \\
& =\left\|x-x^{\prime \prime}\right\|+\left|\left\|x^{\prime \prime}\right\|-\|x\|\right| \leq 2\left\|x-x^{\prime \prime}\right\| \leq \delta
\end{aligned}
$$

This concludes the proof.

Question 2.6. In the previous proof, the constant $\varepsilon_{\phi, \delta}$ depends on $\sigma$ through the dimension $d$ and the constant $M$ from Lemma 2.3. By [12, Theorem 2.6], a discrete Kazhdan group has finitely many unitary irreducible representations of a given finite dimension (up to unitary equivalence), so Theorem 1.3 can be made uniform over all unitary irreducible representations $\sigma$ with dimension less than a given dimension. Can it be made uniform over all finite-dimensional unitary representations? 


\section{Proof of Theorem 1.4}

Let $\left\{U_{n}\right\}$ be a countable basis of open sets in the unit sphere $K$ of $\mathscr{H}$, and let $\Phi$ be the set of all positive definite functions on $G$ defining irreducible finite-dimensional representations. Notice that the set $X^{\prime} \subseteq \operatorname{Rep}(G, \mathscr{H})$ of all representations $\pi$ such that for every $n \in \mathbb{N}$ and every $\delta>0$ there exist $m>0, x \in U_{n}, x_{i} \in K$, $c_{i} \in \mathbb{C} \backslash\{0\}$, and $\phi_{i} \in \Phi, i \leq m$, such that $x=\sum c_{i} x_{i}$, the $x_{i}$ are pairwise orthogonal and each $x_{i}$ is $\left(\pi, \phi_{i}, \varepsilon_{\phi_{i}, \delta_{i}^{\prime}}\right)$-good, where $\delta_{i}^{\prime}=\frac{\delta}{\left|c_{i}\right| \cdot m}$, and $\varepsilon_{\phi_{i}, \delta_{i}^{\prime}}$ is given by Theorem 1.3, is a $G_{\delta}$ set. Indeed, for fixed $n, \delta, m, x, \bar{x}=\left(x_{1}, \ldots, x_{m}\right)$, $\bar{c}=\left(c_{1}, \ldots, c_{m}\right), \bar{\phi}=\left(\phi_{1}, \ldots, \phi_{m}\right)$ as above, the set

$$
V_{x, \bar{x}, \bar{c}, \bar{\phi}}^{n, \delta, m}=\left\{\pi \in \operatorname{Rep}(G, \mathscr{H}): \text { each } x_{i} \text { is }\left(\pi, \phi_{i}, \varepsilon_{\phi_{i}, \delta_{i}^{\prime}}\right) \text {-good }\right\}
$$

is clearly open. We also set $V_{x, \bar{x}}^{n, \bar{c}, \bar{c}, \bar{\phi}}$ to be the empty set if the $x_{i}$ are not pairwise orthogonal or $x \neq \sum c_{i} x_{i}$. Now we can define $X^{\prime}$ by

$$
X^{\prime}=\bigcap_{n \in \mathbb{N}} \bigcap_{\delta \in \mathbb{Q}^{+}} \bigcup_{m \in \mathbb{N}} \bigcup_{x \in U_{n}} \bigcup_{\bar{x} \in K^{m}} \bigcup_{\bar{c} \in \mathbb{C}^{m}} \bigcup_{\bar{\phi} \in \Phi^{m}} V_{x, \bar{x}, \bar{c}, \bar{\phi}}^{n, \bar{s}},
$$

which is a $G_{\delta}$ condition.

Moreover, $X^{\prime}$ is dense in $\operatorname{Rep}(G, \mathscr{H})$ as it contains all direct sums of finitedimensional representations, which, by our assumption, are dense in $\operatorname{Rep}(G, \mathscr{H})$. This is because it is easy to see that for every such sum $\pi$ there are densely many elements $x \in K$ of the form $\sum c_{i} x_{i}$, where $x_{i}$ are pairwise orthogonal unit vectors, and each $x_{i}$ is $\left(\pi, \phi_{i}, \delta\right)$-good for some $\phi_{i}$ and every $\delta>0$.

Now we show that every representation in $X^{\prime}$ is a direct sum of finite-dimensional representations. Fix $\pi \in X^{\prime}$. Using Zorn's lemma, we can decompose $\mathscr{H}$ into $\mathscr{H}_{0}$ and $\mathscr{H}_{1}$ such that $\mathscr{H}_{0}$ is the direct sum of all finite-dimensional representations contained in $\pi$. For $i=0,1$, let $P_{\mathscr{H}_{i}}$ be the orthogonal projection of $\mathscr{H}_{\text {on }} \mathscr{H}_{i}$. Suppose that $\mathscr{H}_{1}$ is not trivial, and fix $0<\delta<1, x \in K$, pairwise orthogonal $x_{i} \in K$ and $c_{i} \in \mathbb{C} \backslash\{0\}, i \leq m$, such that $x=\sum c_{i} x_{i}$, each $x_{i}$ is $\left(\pi, \phi_{i}, \varepsilon \phi_{i}, \frac{\delta}{\left|c_{i}\right| . m}\right.$ )-good for some $\phi_{i} \in \Phi$, and $\left\|x-P_{\mathcal{H}_{0}} x\right\|>\delta$ (the last condition can be satisfied by choosing $x$ in an appropriate $U_{n}$ ): By Theorem 1.3, there exist $x_{i}^{\prime} \in K, i \leq m$, inducing irreducible finite-dimensional representations, and such that

$$
\left\|x_{i}-x_{i}^{\prime}\right\|<\frac{\delta}{\left|c_{i}\right| . m}
$$

that is, $\left\|x-\sum c_{i} x_{i}^{\prime}\right\|<\delta$. But then, clearly, $x_{i_{0}}^{\prime} \notin \mathscr{H}_{0}$ for some $i_{0} \leq m$, as if this were not the case, we would get that

$$
\left\|x-\sum c_{i} x_{i}^{\prime}\right\| \geq\left\|x-P_{\mathscr{H}_{0}} x\right\|>\delta .
$$

Since $P_{\mathscr{H}_{1}}$ is a $G$-intertwiner, the image under $P_{\mathscr{H}_{1}}$ of the linear span of $\pi(G) x_{i_{0}}^{\prime}$, is an invariant subspace of $\mathscr{H}_{1}$, which is a contradiction. 
Now let $X^{\prime \prime}$ be the set of all representations that contain every finite-dimensional representation with infinite multiplicity. As $G$ is a Kazhdan group, we can see that $X^{\prime \prime}$ is given by a $G_{\delta}$ condition. Indeed, for $[\sigma]$ the isomorphism class of a finite-dimensional unitary irreducible representation of $G$, and $n>0$, let $V_{[\sigma], n}$ be the set of representations $\pi \in \operatorname{Rep}(G, \mathcal{H})$ such that $[\sigma]$ appears in $\pi$ with multiplicity at least $n$. Clearly, $V_{[\sigma], n}$ is open and $X^{\prime \prime}=\bigcap_{[\sigma]} \bigcap_{n} V_{[\sigma], n}$, where the intersection is countable because there are countably many $[\sigma]$.

By our assumption on $C^{*}(G)$, the set $X^{\prime \prime}$ is dense. Thus, $X=X^{\prime} \cap X^{\prime \prime}$ is a dense $G_{\delta}$ set, all the representations of which are direct sums of finite-dimensional representations, each appearing with infinite multiplicity. Clearly, all elements in $X$ are conjugate.

Remark 3.1. The converse of Theorem 1.4 also follows from [9, Theorem 2.5]. That is, if either $G$ does not have Property (T), or $C^{*}(G)$ is not residually finitedimensional, then all classes in $\operatorname{Rep}(G, \mathscr{H})$ are meager. We will now prove this. By [9, Theorem 2.5], if for a separable $C^{*}$-algebra $A$ the set of isolated points in $\hat{A}$ is not dense, then the restriction of the action of $U(\mathscr{H})$ by conjugation on a dense $G_{\delta}$ invariant subset of $\operatorname{Rep}(A, \mathscr{H})$ is turbulent. That, by the definition of turbulence, in particular implies that every class in $\operatorname{Rep}(G, \mathscr{H})$ is meager. Now take $A=C^{*}(G)$ and notice that isolated points in $\hat{G}$ correspond to finite-dimensional representations. Indeed, suppose that $A$ is an arbitrary unital separable $C^{*}$-algebra and $\pi \in \hat{A}$ is isolated. Then we can decompose $A$ as $I \oplus J$, where $\hat{J}=\{\pi\}$. As $J$ is a separable $C^{*}$-algebra with a one-point spectrum, it is isomorphic to the compact operators on the Hilbert space $\mathscr{H}_{\pi}$. Since $A$ is unital, so is $J$, meaning that $J$ and $\pi$ are finite-dimensional. It follows that when $G$ does not have Property (T), $\hat{G}$ does not have isolated points, by [12, Theorem 2.1]; when $C^{*}(G)$ is not residually finite-dimensional, then the isolated points in $\hat{G}$ are not dense by Archbold's main result in [1].

\section{Proof of Theorem 1.5}

For a unitary representation $\pi$, we denote by $\infty \cdot \pi$ the $\ell^{2}$-direct sum of countably many copies of $\pi$.

Lemma 4.1. Let $H$ be a locally compact group. Assume that $H$ has (up to unitary equivalence) countably many finite-dimensional irreducible unitary representations $\sigma_{1}, \sigma_{2}, \ldots$. Then the representation $\bigoplus_{n=1}^{\infty} \infty \cdot \sigma_{n}$ is unitarily equivalent to a Koopman representation.

Proof. View $\sigma_{n}$ as a continuous homomorphism $H \rightarrow U\left(N_{n}\right)$. Let $K_{n}$ denote the closure of $\sigma_{n}(H)$ in $U\left(N_{n}\right)$, so that $K_{n}$ is a compact group (on which $H$ acts by 
left translations by elements of $\left.\sigma_{n}(H)\right)$. Let $m_{n}$ denote normalized Haar measure on $K_{n}$, and let $\lambda_{n}$ denote the regular representation of $K_{n}$ on $L^{2}\left(K_{n}, m_{m}\right)$. For $p \geq 1$, let $K_{n, p}$ denote a copy of $K_{n}$ endowed with the measure $2^{-n-p} m_{n}$. Set $X=\bigsqcup_{n, p} K_{n, p}$, endowed with the $H$-invariant probability measure

$$
\mu=\bigoplus_{n, p} 2^{-n-p} m_{n} .
$$

Note that the $H$-representations on $L^{2}(X, \mu)$ and on $L_{0}^{2}(X, \mu)$ are equivalent, as $L^{2}(X, \mu)$ contains the trivial representation with infinite multiplicity.

So it is enough to prove that the $H$-representation $\pi$ on $L^{2}(X, \mu)$ is equivalent to $\bigoplus_{n=1}^{\infty} \infty \cdot \sigma_{n}$. To see this, first observe that $\pi$ is equivalent to $\bigoplus_{n} \infty \cdot \pi_{n}$, where $\pi_{n}=\lambda_{n} \circ \sigma_{n}$. By the Peter-Weyl theorem, $\pi_{n}$ decomposes as a direct sum of finite-dimensional irreducible representations of $H$, hence of certain representations $\sigma_{k}$, and moreover $\sigma_{n}$ is a sub-representation of $\pi_{n}$ (because the natural representation of $K_{n}$ on $\mathbb{C}^{N_{n}}$ is irreducible, hence appears as a sub-representation of $\lambda_{n}$ ). This shows that $\bigoplus_{n} \infty \cdot \pi_{n}$ is equivalent to $\bigoplus_{n} \infty \cdot \sigma_{n}$.

To prove Theorem 1.5, observe that a discrete Kazhdan group $G$ satisfies the assumption of Lemma 4.1 (by [12, Theorem 2.6]). Let $\left(\sigma_{n}\right)_{n \in \mathbb{N}}$ be an enumeration of all finite-dimensional irreducible unitary representations of $G$. By Theorem 1.4 and its proof, the representation $\bigoplus_{n=1}^{\infty} \infty \cdot \sigma_{n}$ has a comeager conjugacy class.

In particular, we get the following statement which was proved in [4] for finite abelian groups.

Corollary 4.2. Let $G$ be a finite group. Then the set of unitary representations realizable by an action is comeager in $\operatorname{Rep}(G, \mathscr{H})$.

Remark 4.3. Kechris proves (see [8, Section (F) in Appendix H]) that, if $G$ is torsion-free abelian, then the set of representations realizable by an action is meager in $\operatorname{Rep}(G, \mathscr{H})$.

\section{Bibliography}

[1] R. J. Archbold, On residually finite-dimensional $C^{*}$-algebras, Proc. Amer. Math. Soc. 123 (1995), no. 9, 2935-2937.

[2] B. Bekka, On the full $C^{*}$-algebras of arithmetic groups and the congruence subgroup problem, Forum Math. 11 (1999), no. 6, 705-715.

[3] B. Bekka, P. de la Harpe and A. Valette, Kazhdan's Property (T), New Math. Monogr. 11, Cambridge University Press, Cambridge, 2008.

[4] M. Doležal, Unitary representations of finite abelian groups realizable by an action, Topology Appl. 164 (2014), 87-94. 
[5] M. Ershov and A. Jaikin-Zapirain, Groups of positive weighted deficiency and their applications, J. Reine Angew. Math. 677 (2013), 71-134.

[6] R. Exel and T. A. Loring, Finite-dimensional representations of free product $C^{*}$-algebras, Internat. J. Math. 3 (1992), no. 4, 469-476.

[7] A. S. Kechris, Classical Descriptive Set Theory, Grad. Texts in Math. 156, Springer, New York, 1995.

[8] A. S. Kechris, Global Aspects of Ergodic Group Actions, Math. Surveys Monogr. 160, American Mathematical Society, Providence, 2010.

[9] D. Kerr, H. Li and M. Pichot, Turbulence, representations, and trace-preserving actions, Proc. Lond. Math. Soc. (3) 100 (2010), no. 2, 459-484.

[10] A. Lubotzky and Y. Shalom, Finite representations in the unitary dual and Ramanujan groups, in: Discrete Geometric Analysis, Contemp. Math. 347, American Mathematical Society, Providence (2004), 173-189.

[11] V. G. Pestov and V. V. Uspenskij, Representations of residually finite groups by isometries of the Urysohn space, J. Ramanujan Math. Soc. 21 (2006), no. 2, 189-203.

[12] P. S. Wang, On isolated points in the dual spaces of locally compact groups, Math. Ann. 218 (1975), no. 1, 19-34.

[13] R. J. Zimmer, Ergodic Theory and Semisimple Groups, Monogr. Math. 81, Birkhäuser, Basel, 1984.

Received December 22, 2017; revised July 19, 2018.

\section{Author information}

Michal Doucha, Institute of Mathematics, Czech Academy of Sciences,

Žitná 25, 11567 Praha 1, Czech Republic.

E-mail: doucha@math.cas.cz

Maciej Malicki, Department of Mathematics and Mathematical Economics,

Warsaw School of Economics, al. Niepodległości 162, 02-554 Warsaw, Poland.

E-mail: mamalicki@gmail.com

Alain Valette, Institut de Mathématiques, Université de Neuchâtel,

Unimail, 11 Rue Emile Argand, 2000 Neuchâtel, Switzerland.

E-mail: alain.valette@unine.ch 